\title{
Refractive outcomes and safety of the implantable collamer lens in young low-to-moderate myopes
}

\author{
This article was published in the following Dove Press journal: \\ Clinical Ophthalmology \\ 31 January 2017 \\ Number of times this article has been viewed
}

\author{
Paul J Dougherty ${ }^{1,2}$ \\ Taylor Priver' \\ 'Dougherty Laser Vision, Westlake \\ Village, CA, USA; '2Jules Stein Eye \\ Institute at the David Geffen School \\ of Medicine at UCLA, Los Angeles, \\ CA, USA
}

Video abstract

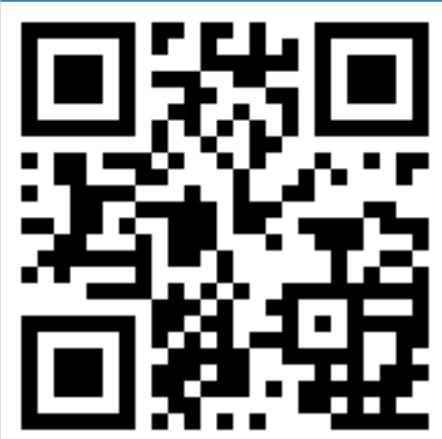

Point your SmartPhone at the code above. If you have a QR code reader the video abstract will appear. Or use: http://youtu.be/hj[RT9nr]x]

Correspondence: Paul J Dougherty Dougherty Laser Vision, 4353 Park Terrace Drive \#I50, Westlake Village, CA 91361, USA

Tel +l 8059875300

Email natasha@doughertylaservision.com
Purpose: To evaluate visual and refractive outcomes and safety of implantable collamer lens (ICL) implantation in low-to-moderate myopia.

Setting: Private Practice - Dougherty Laser Vision, Westlake Village, CA, USA.

Methods: A chart review was performed in 56 (104 eyes) consecutive patients who underwent implantation of the Visian ICL V4 model. Inclusion criteria were age between 21 and 40 years, spherical equivalent between $-3.00 \mathrm{D}$ and $-10.00 \mathrm{D}$, and cylinder up to $-5 \mathrm{D}$. Data on visual acuity, refraction, and complications were collected.

Results: The mean time to the last postoperative visit was $13.1 \pm 14.0$ months (range, 2 to 50 months). The mean preoperative spherical equivalent was $-6.96 \pm 1.60 \mathrm{D}$ and the mean preoperative cylinder was $-1.03 \pm 0.88 \mathrm{D}$. The mean preoperative corrected distance visual acuity (CDVA) was 20/20 (range, 20/15 to 20/80) that remained unchanged at last postoperative visit. At the last postoperative visit, the spherical equivalent was $-0.08 \pm 0.01 \mathrm{D}$ and the cylinder was $0.29 \pm 0.71 \mathrm{D}$. Comparison of postoperative uncorrected distance visual acuity (UCDVA) to preoperative CDVA indicated that 27 eyes (26\%) had better postoperative UCDVA and 61 eyes (59\%) had UDVA equivalent to the preoperative CDVA. Postoperatively, 4 eyes lost 1 line of CDVA and no eyes lost $>1$ line of CDVA. There were 32 eyes that gained at least $\geq 1$ of CDVA. There were no intraoperative complications. Postoperatively, 3 patients ( 6 eyes) had dry eye, and 1 patient complained of nighttime halos in 1 eye. At the last postoperative visit, there were no eyes with lens opacity or glaucoma.

Conclusion: ICL implantation for low and moderate myopia up to $-10 \mathrm{D}$ in patients up to 40 years of age was safe, accurate, and efficacious. ICL implantation may be a good alternative to laser in situ keratomileusis for young myopes less than $-10.00 \mathrm{D}$.

Keywords: ICL, low myopia, Costa Rica, Dougherty Laser Vision

\section{Introduction}

Historically, the implantable collamer lens (ICL) has been used for the treatment of high myopia due to the decreased efficacy and safety of laser in situ keratomileusis (LASIK) and photorefractive keratectomy (PRK) in these patients. Theoretical comparisons of LASIK and ICL implantation for high refractive error indicate better optical and visual quality after ICL implantation. ${ }^{1}$ Comparisons of LASIK and ICL implantation for high myopia indicate essentially equivalent refractive outcomes. ${ }^{2}$ A recent comparison of wavefront-guided LASIK and ICL implantation in high myopes indicated better visual performance in eyes that underwent ICL implantation. ${ }^{3}$ Schallhorn et $\mathrm{al}^{4}$ demonstrated superior visual, refractive, and safety outcomes with the toric ICL (TICL) compared to PRK for moderate and high myopic astigmatism.

However, low-to-moderate myopes may be better candidates for ICL implantation due to pupil size, dry eyes, inadequate tissue volume for LASIK or abnormal 
topographic shape. Alternately, patients may have a personal preference for a reversible procedure (ICL implantation) with better visual quality due to the lack of induced higher-order aberrations, ${ }^{5}$ versus a permanent procedure (LASIK) where corneal shape is altered and dry eye may be induced. The Visian ICL (STAAR Surgical Co., Monorovia, CA, USA) was granted approval by the United States Food and Drug Administration (FDA) in late 2005 for spherical myopia of -3.00 to $-20.00 \mathrm{D}$. In the current study, the visual and refractive outcomes and safety of ICL implantation were investigated in low-to-moderate myopia.

\section{Methods}

This retrospective study was performed by chart review of 56 (104 eyes) consecutive patients with low-to-moderate myopia who underwent ICL implantation by a single surgeon (PJD). Inclusion criteria were age between 21 and 40 years (Table 1), myopia between -3.00 and $-10.00 \mathrm{D}$ (spherical equivalent), cylinder up to $-5 \mathrm{D}$, no preexisting ocular pathology or previous eye surgery, no systemic disease, anterior chamber depth (ACD) of $\geq 2.60 \mathrm{~mm}$ (measured from the corneal endothelium to the anterior lens capsule), and endothelial cell count $>2,000$ cells $/ \mathrm{mm}^{2}$.

A thorough ophthalmic examination was performed including measurement of uncorrected distance visual acuity (UCDVA) and corrected distance visual acuity (CDVA) using a Snellen chart, manifest and cycloplegic refractions, corneal topography, central pachymetry, applanation tonometry, horizontal corneal diameter (white-to-white), anterior segment ultrasonic biomicroscopy (UBM) for sulcus to sulcus diameter, slit lamp biomicroscopy, ACD, funduscopy, and corneal endothelial cell count (cells $/ \mathrm{mm}^{2}$ ). A thorough written and verbal informed consent was obtained by the surgeon from each patient prior to surgery. Patient informed consent to participate in this retrospective study was obtained in writing prior to surgery. Approval was sought from St Vincent's Hospital institutional review board in Los Angeles, CA, and was deemed not needed.

Examinations were performed preoperatively and postoperatively at 4 hours, 1 day, 1, 3, 6, and 12 months, and yearly thereafter. All eyes were targeted for emmetropia except

Table I Preoperation vs postoperation data

\begin{tabular}{lll}
\hline Variable & Preoperation & Postoperation \\
\hline Age range, years & $21-40$ & $21-40$ \\
Myopia, D & $-6.96 \pm 1.60$ & $-0.08 \pm 0.01$ \\
Astigmatism, D & $1.03 \pm 0.88$ & $0.29 \pm 0.7 \mathrm{I}$ \\
UCDVA (\% 20/20 or better) & $0 \%$ & $78 \%$ \\
\hline
\end{tabular}

Abbreviation: UCDVA, uncorrected distance visual acuity. for 1 patient who requested monovision and was targeted for $-0.75 \mathrm{D}$ in 1 eye.

The ICL is a plate-haptic single-piece lens designed for implantation in the posterior chamber. The ICL is made of collamer, which is a flexible, hydrophilic material derived from collagen that is a copolymer comprising hydrophilic collagen and hydroxyethyl methacrylate (HEMA). The lens has a central convex-concave optic zone with a diameter of 4.9-5.8 mm, depending on dioptric power. The ICL is $6.0 \mathrm{~mm}$ wide with diameters of $12.1,12.6,13.2$, and $13.7 \mathrm{~mm}$. In the current study, all patients underwent implantation of the ICL V4 model. Lens power calculations were performed based on formulas supplied by the manufacturer. Sizing calculations before 2006 performed with the Staar modified FDA sizing nomogram using white-to-white diameter as an estimate of sulcus size. Sizing calculations starting in 2006 were performed with the VuMax UBM (Sonomed, Lake Success, NY, USA) unit to measure sulcus-to-sulcus using the Sonomed nomogram. ${ }^{6}$ Thirty eyes underwent limbal relaxing incisions (LRIs) during the ICL implantation procedure for the treatment of visually significant astigmatism.

\section{Statistical analysis}

Data were collected on visual acuity, refractive outcomes, and complications at each postoperative visit. Visual acuity data were converted to logarithm of the minimum angle of resolution (LogMAR) values for statistical analysis. Data are presented for the preoperative visit and the last postoperative visit. The mean and standard deviation values are presented here. Statistical analysis was performed with Excel (Microsoft Corp., Redmond, WA, USA).

\section{Results}

The mean preoperative spherical equivalent was $-6.96 \pm 1.60 \mathrm{D}$ and the mean preoperative cylinder was $1.03 \pm 0.88 \mathrm{D}$. The mean preoperative CDVA was 20/20 (range, 20/15 to 20/80). The last postoperative visit ranged from 2 months postoperatively to 50 months postoperatively (mean $13.1 \pm 14.1$ months).

At the last postoperative visit, the spherical equivalent was $-0.08 \pm 0.01 \mathrm{D}$ and the cylinder was $0.29 \pm 0.71 \mathrm{D}$ (Figure 1 and Table 1). The mean postoperative CDVA was 20/20 (range, 20/15 to 20/80) (Figure 2). The refracting technician mistakenly neglected to record the CDVA of 1 patient (2 eyes).

Postoperative UCDVA is presented in Figure 3 and Table 1. Comparison of postoperative UCDVA to preoperative CDVA indicated that 27 eyes $(26 \%)$ had better postoperative UCDVA and that 61 eyes (59\%) had UCDVA 


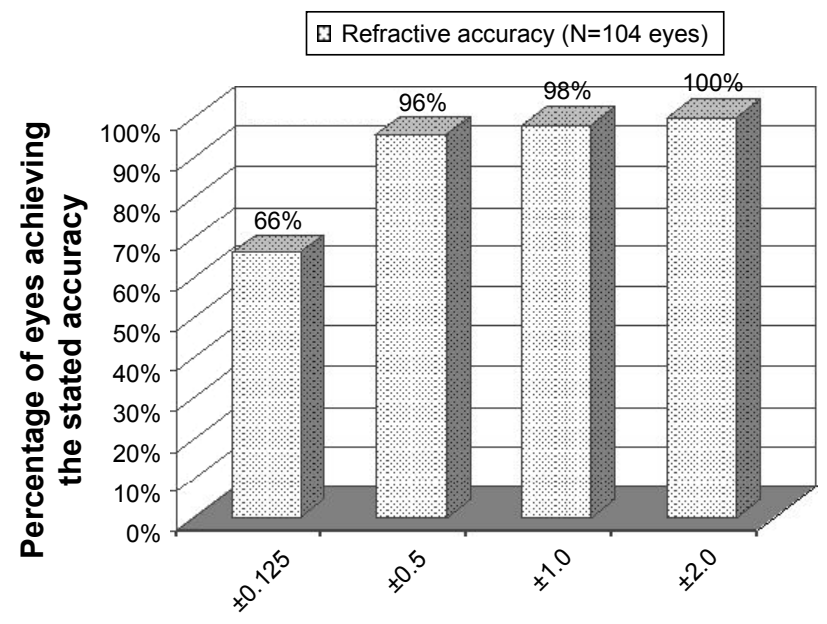

Figure I Refractive outcome percent within attempted at last postoperative visit of eyes with low-to-moderate myopia that underwent implantable collamer lens implantation.

equivalent to the preoperative CDVA. Postoperatively, 4 eyes lost 1 line of CDVA and no eyes lost more than 1 line of CDVA (Figure 4). There were 32 eyes that gained at least 1 or more lines of CDVA and no eyes gained $>2$ lines of CDVA (Figure 4).

There were no intraoperative complications. Postoperatively, 3 patients ( 6 eyes) had dry eye, 2 with mild symptoms, and 1 with moderate symptoms. All 3 patients were managed with topical lubricant therapy. One patient ( 2 eyes) reported unclear vision despite Snellen acuity of 20/20. One patient complained of halos at night in 1 eye only and was managed with Alphagan-P (brimonidine tartrate ophthalmic solution; Allergan Inc., Irvine, CA, USA) as required. Residual refractive error was treated with LASIK (14 eyes), PRK ( 3 eyes), and LRI ( 2 eyes) 3 months after ICL implantation.

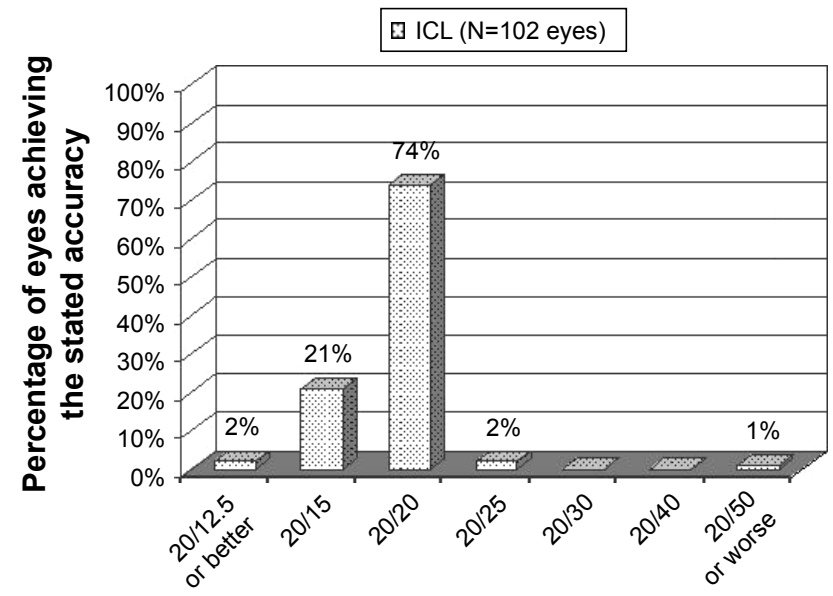

Figure 2 Corrected distance visual acuity at last visit of eyes with low-to-moderate myopia that underwent implantable collamer lens implantation. Abbreviation: ICL, implantable collamer lens.

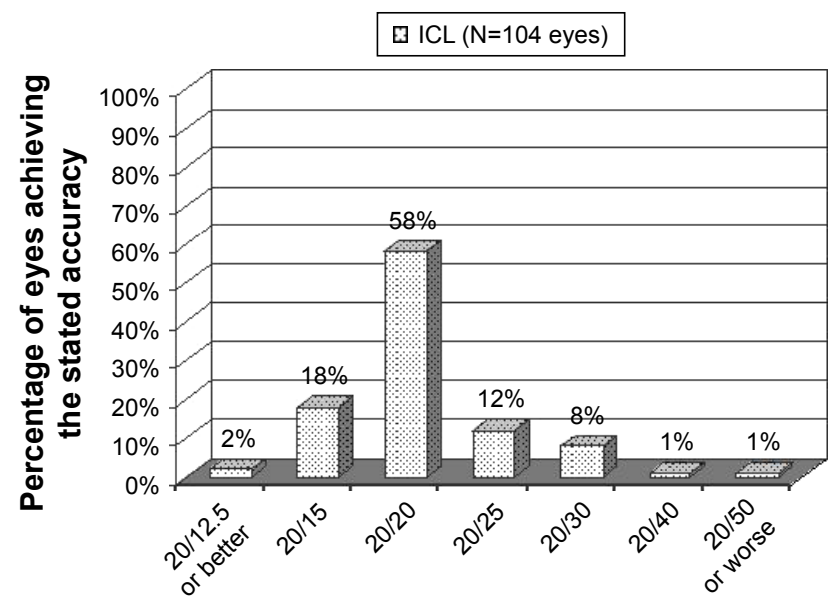

Figure 3 Uncorrected distance visual acuity at last visit of eyes with low-tomoderate myopia that underwent implantable collamer lens implantation.

Abbreviation: ICL, implantable collamer lens.

At the last postoperative visit, no eye was noted to have any lens opacity or glaucoma.

\section{Discussion}

The outcomes of this study of the ICL for low-to-moderate myopia indicate that the ICL is a safe and effective treatment for correction in the range that is normally reserved for LASIK. The postoperative UCDVA was the same or better than preoperative BCVA in $85 \%$ (88 of 104) of eyes. This outcome indicates excellent efficacy of ICL implantation. Fast visual recovery, usually within 24 hours, was observed which remained stable over the postoperative period.

In the current study, $96 \%$ of eyes were within $\pm 0.50 \mathrm{D}$ of emmetropia. This outcome is better than the outcomes

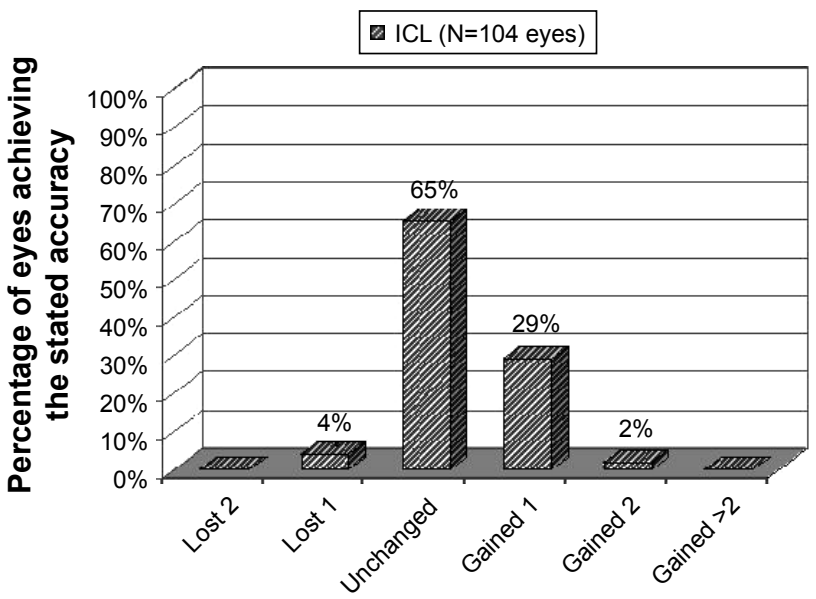

Figure 4 Change in corrected distance visual acuity at last postoperative visit of eyes with low-to-moderate myopia that underwent implantable collamer lens implantation.

Abbreviation: ICL, implantable collamer lens. 
reported for the treatment of low myopia with recent studies of ICL as well as standard, wavefront-optimized, and wavefront-guided LASIK. For example, Sanders ${ }^{7}$ reported that $85 \%$ of eyes with preoperative spherical equivalent between -3 and $-7.88 \mathrm{D}$ were within $\pm 0.50 \mathrm{D}$ after ICL implantation compared to only $67 \%$ in the LASIK group 6 months after surgery $(P=0.002)$. Differing enrollment criteria or incorporating data from multiple surgeons in the Sanders ${ }^{7}$ study may explain the difference between studies. The accuracy of the outcomes of the current study is also better than recent studies of aspheric LASIK that reported $87 \%$ of eyes within $\pm 0.50 \mathrm{D}^{8}$ and a meta-analysis of wavefront-guided LASIK by Fares et al that demonstrated that $91.8 \%$ of eyes were within \pm 0.50 D. ${ }^{9}$ Sanders and Sanders reported that while TICL and custom LASIK had comparable clinical efficacy, TICL had a significantly better postoperative improvement in best spectacle corrected visual acuity and significantly better postoperative UCDVA than preoperative best spectacle corrected visual acuity than custom LASIK. ${ }^{10}$ The accuracy in the present study implies that most ICL patients will not require enhancement with LASIK or PRK to achieve the targeted refraction following the initial surgery.

Postoperative CDVA in the current study was well within the range reported for ICL and LASIK. A multisite FDA study of the TICL for myopic astigmatism reported $97 \%$ of eyes with 20/20 or better CDVA at 12 months postoperatively. ${ }^{11}$ This is similar to the result of $97 \%$ of eyes with CDVA of 20/20 or better at last visit. A recent comparison of aspheric, wavefront-optimized, and wavefront-guided LASIK with 3 excimer laser platforms reported mean CDVA ranging from $\sim 20 / 40$ to $20 / 20 .{ }^{12}$ Similarly, mean CDVA of 20/20 was determined.

Data from the FDA trial of the ICL for moderate and high myopia reported improved CDVA with few complications. ${ }^{13}$ The safety profile of any refractive procedure is evaluated based on the postoperative loss or gain of corrected distance visual acuity compared to preoperatively. It was found that only $4 \%$ of eyes lost 1 line of CDVA (typically due to dry eye) and no eyes lost $>1$ line. In fact, a much higher rate of improved CDVA (31\% gained 1 or more lines) compared to preoperatively was observed. These outcomes indicate an excellent safety profile for ICL implantation in the cohort of patients treated in this study. Additionally, other complications were rare and managed medically. No patients required explanation of ICL or other surgical procedures. Low complication rates have been previously reported with the ICL. ${ }^{11,14}$
Last, many surgeons do not offer ICL to low and moderate myopes due to the theoretic increased risk of premature cataract formation. In the FDA study, the cohort of eyes with 5-year follow-up had a $1.3 \%$ rate of premature cataract formation requiring surgery. In this cohort, every patient who developed premature was $>40$ years of age (except 1 patient) and had myopia of $-13.00 \mathrm{D}$ or more. ${ }^{15}$ Alfonso et al found that only 21 of $3,420(0.61 \%)$ Visian ICL eyes developed visually significant cataract a mean of 4.2 years after implantation. ${ }^{16}$ Lisa et $\mathrm{al}^{17}$ found a $0 \%$ incidence of cataract formation in a 1-year follow-up of Visian ICL for myopia. In the current study of young low myopes aged $\leq 40$ years and $<-10.00$, a rate of premature cataract formation was $0 \%$, implying a high level of safety in this group of patients.

In summary the ICL implantation for low and moderate myopia up to $-10 \mathrm{D}$ in patients aged $\leq 40$ years, was accurate, efficacious, and safe. The results of this study were slightly better than previously published studies of wavefront-guided and wavefront-optimized LASIK, implying that ICL is a good alternative to LASIK in this group of patients.

\section{Disclosure}

Dr Dougherty is a paid consultant to Staar Surgical. The authors report no other conflicts of interest in this work.

\section{References}

1. Pérez-Vives C, Dominguez-Vicent A, García-Lázaro S, Ferrer-Blasco T, Montés-Micó R. Optical and visual quality comparison of implantable collamer lens and laser in situ keratomileusis for myopia using an adaptive optics visual simulator. Eur J Ophthalmol. Epub 2012 Jul 30.

2. Barsam A, Allan BD. Excimer laser refractive surgery versus phakic intraocular lenses for the correction of moderate to high myopia. Cochrane Database Syst Rev. 2014(6):CD007679.

3. Kamiya K, Igarashi A, Shimizu K, Matsumura K, Komatsu M. Visual performance after posterior chamber phakic intraocular lens implantation and wavefront-guided laser in situ keratomileusis for low to moderate myopia. Am J Ophthalmol. 2012;153(6):1178-1186.

4. Schallhorn S, Tanzer D, Sanders DR, Sanders ML. Randomized prospective comparison of visian toric implantable collamer lens and conventional photorefractive keratectomy for moderate to high astigmatism. $J$ Refract Surg. 2007;23(9):853-867.

5. Dougherty PJ, Trueb P, Bains HS. Higher order aberrations after Visian ICL implantation. Poster presented at: The European Society of Cataract and Refractive Surgery Annual Meeting; September 2008; Berlin, Germany.

6. Dougherty PJ, Rivera RP, Schneider D, Lane SS, Brown D, Vukich J. Improving accuracy of phakic IOL (Visian ICL - Staar Surgical) sizing utilizing high frequency ultrasound (UBM) with the Sonomed Vumax II. $J$ Cataract Refract Surg. 2010;37(1):13-18.

7. Sanders D. Matched population comparison of the visian implantable collamer lens (ICL) and standard LASIK for myopia of -3.00 to -7.88 diopters. J Refract Surg. 2007;23:537-552.

8. Woodcock M, Shah S, Mandal N, Pieger S, Grills C, Moore TC. Small optical zones with aspheric profiles in laser refractive surgery for myopia: a surgical outcome and patient satisfaction study. Cont Lens Anterior Eye. 2013;36(5):259-264. 
9. Fares U, Suleman H, Al-Aqaba MA, Otri AM, Said DG, Dua HS. Efficacy, predictability, and safety of wavefront-guided refractive laser treatment: metaanalysis. J Cataract Refract Surg. 2011;37(8): 1465-1475.

10. Sanders DR, Sanders ML. Comparison of the toric implantable collamer lens and custom ablation LASIK for myopic astigmatism. J Refract Surg. 2008;24:773-778.

11. Sanders DR, Schneider D, Martin R, et al. Toric implantable collamer lens for moderate to high myopic astigmatism. Ophthalmology. 2007; 114(1):54-61.

12. Han DC, Chen J, Htoon HM, Tan DT, Mehta JS. Comparison of outcomes of conventional WaveLight $\left({ }^{\circledR}\right)$ Allegretto Wave $\left({ }^{\circledR}\right)$ and Technolas $\left({ }^{\circledR}\right)$ excimer lasers in myopic laser in situ keratomileusis. Clin Ophthalmol. 2012;6:1159-1168.

13. Sanders DR, Brown DC, Martin RG, Shepherd J, Deitz MR, DeLuca M. Implantable contact lens for moderate to high myopia: phase I FDA clinical study with 6 month follow-up. J Cataract Refract Surg. 1998;24: 607-611.
14. Zaldivar R, Davidorf JM, Oscherow S. Posterior chamber phakic intraocular lens for myopia of -8 to -19 diopters implantation. J Refract Surg. 1998;14:294-305.

15. Sanders DR. Anterior subcapsular opacities and cataracts 5 years after surgery in the visian implantable collamer lens FDA trial. J Refract Surg. 2008;24(6):566-570.

16. Alfonso JF, Lisa C, Fernández-Vega L, Almanzar D, Pérez-Vives C, Montés-Micó R. Prevalence of cataract after collagen copolymer phakic intraocular lens implantation for myopia, hyperopia, and astigmatism. $J$ Cataract Refract Surg. 2015;41(4):800-805.

17. Lisa C, Alfonso JF, Alfonso-Bartolozzi B, Fernández-Vega L, Pérez-Vives C, Montés-Micó R. Collagen copolymer posterior chamber phakic intraocular lens supported by the ciliary sulcus to treat myopia: one-year follow-up. J Cataract Refract Surg. 2015;41(1):98-104.
Clinical Ophthalmology

\section{Publish your work in this journal}

Clinical Ophthalmology is an international, peer-reviewed journal covering all subspecialties within ophthalmology. Key topics include: Optometry; Visual science; Pharmacology and drug therapy in eye diseases; Basic Sciences; Primary and Secondary eye care; Patient Safety and Quality of Care Improvements. This journal is indexed on

Submit your manuscript here: http://www.dovepress.com/clinical-ophthalmology-journal

\section{Dovepress}

PubMed Central and CAS, and is the official journal of The Society of Clinical Ophthalmology (SCO). The manuscript management system is completely online and includes a very quick and fair peer-review system, which is all easy to use. Visit http://www.dovepress.com/ testimonials.php to read real quotes from published authors. 